\title{
BEAM-BEAM INTERACTION AND HIGH ORDER RESONANCES
}

\section{S. Tepikian and S.Y. Leet}

Brookhaven National Laboratory

Upton, NY 11973, USA
BNL- -45522

DE91 014034

\section{Abstract}

There is experimental evidence from SPS[1], that very high order resonances (an order $>10$ ) can cause particle loss during the beam-beam interaction. These results can be simulated by tracking a single particle against a round beam. If the round bearn has an rms sire of $\sigma$ and the particle's initial amplitude is $2 \sigma$ then no effects are observed due to high order resonances. However, if the particle's initial amplitude is $5 \sigma$ the beambeam 16th order resonances are strong. Furthermore, if we add tune modulation (i.e. power supply ripple) then the $5 \sigma$ particle's motion becomes chaotic (when it is near a 16th order resonance) and can result in particle loss. This is in agreement with the beam-ream experiments performed at the SPS. Implications on RHIC and SSC will be discussed.

\section{INTRODUCTION}

Beam-beam experiments on the SPS have shown that when colliding a proton beam $(\approx 26 \pi \mathrm{mm} \cdot \mathrm{mrad})$ against an anti-proton beam ( $\approx 13 \pi \mathrm{mm} \cdot \mathrm{mrad})$, there is an increase in background proton radiation when the tunes cross through the 16th order resonance. One surprise is that the loss oecurred to the larger beam. The reason for this will be made clear as we continue.

Beam-beam effects have been studied both experimentally and theoretically.[2-8] However, most of these studies dealt with the low order resonance effects (an order $<10$ ). In order to study the high order resonance effects we tracked a single particle, with a given initial amplitude, through a collision point against a round beam of rms radius $\sigma$. The low order resonances were avoided by choosing the operating tunes of RHIC. [9]

\section{THE SIMULATION}

The main accelerator is modeled as a linear machine with the following transfer matrix

$$
\left(\begin{array}{cccc}
\cos 2 \pi \nu_{x} & \beta_{x}^{*} \sin 2 \pi \nu_{x} & 0 & 0 \\
-\frac{1}{\sin \sin 2 \pi \nu_{x}} & \cos 2 \pi \nu_{x} & 0 & 0 \\
0 & 0 & \cos 2 \pi \nu_{y} & \beta_{y}^{*} \sin 2 \pi \nu_{y} \\
0 & 0 & -\frac{1}{\beta_{y}} \sin 2 \pi \nu_{y} & \cos 2 \pi \nu_{y}
\end{array}\right)
$$

-Work performed under the auppices of the U.S. Department of Energy.

tPresent sddres: Dept. of Physica, Indiana University, Bloomington, IN 47405. where $\beta_{x}^{*}$ and $\beta_{y}^{*}$ are beta values at the crossing point and $\nu_{x}, \nu_{y}$ are horizontal and vertical-betation tunes respectively.

After the particle circles the accelerator, it will encounter the round beam at the crossing point. This beam-beam interaction is modeled with the following potential

$$
U(r)=\frac{N r_{0}}{2 \gamma \sigma} V(r)=\frac{N r_{0}}{2 \gamma \sigma} e^{-r^{2} / 2 \sigma}
$$

where $N$ is the number of particles, $r_{0}=q^{2} e^{2} / A m_{0} c^{2}$ is the classical radius of the particle with charge qe, mass number $A, m_{0}$ is the atomic mass unit, $\gamma$ is the Lorentz factor and $\sigma$ is the rms beam size. From the above potential, the kicks to the slope become:

$$
\begin{aligned}
& \Delta x^{\prime}=4 \pi \xi f(r) \frac{x}{\beta^{*}} \\
& \Delta y^{\prime}=4 \pi \xi f(r) \frac{y}{\beta^{*}}
\end{aligned}
$$

where $\xi=N r_{0} \beta^{*} / 4 \pi \gamma \sigma^{2}$ is the linear beam-beam parametes, $\beta^{*}=\beta_{x}^{*}=\beta_{y}^{*}$ and

$$
f(r)=\frac{2 \sigma^{2}}{r^{2}}\left[1-e^{-r^{2} / 2 \sigma^{2}}\right] \underset{r \rightarrow 0}{\sim} 1 .
$$

A particle with an initial maximum amplitude of $x_{0}=y_{0}=2 \sigma$ will have an rms amplitude over betatron motion of $<x\rangle_{r m a}=\langle y\rangle_{r m i}=\sqrt{2} \sigma$. Hence, the particle is most likely to be found in a box of area $8 \sigma^{2}$. A particle with the same probabilitiea in a circle has a radius, $r=\sqrt{\frac{\delta}{\pi}} \sigma$, which occupies the same area. Expanding the potential term into a multipole expansion leaves

$$
V(r)=\sum_{k=0} \frac{1}{k !}\left(-\frac{r^{2}}{2 \sigma^{2}}\right)^{2}
$$

The multipoles in this case are very small. However, when the initial maximum amplitude is $x_{0}=y_{0}=5 \sigma$, the corresponding radius is $r=5 \sqrt{\frac{3}{z}} \sigma$. This leads to large multipole terms, in particular the 8th term (driving the 16th order resonance) grow to $\sim 400$ and the 11 th term (driving the 22nd order resonance) then drope to $\sim 200$. This is confirmed with tracking diecumed in section III.

When two beams are colliding with different beam sizes, particles of the larger beam are more likely to be at the $5 \sigma$ position than thoee of the smaller beam, then the losses will be seen in the larger beam. Additionally,

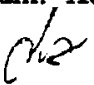


only even order resonances are excited by this multipole expansion. However, when two beams are colliding off center, the odd order resonances will become important.

The final ingredient in the simulation is to include the linear effects of the beam-beam potential. This affects both the betatron tunes and the $\beta^{*}$ at the crossing point. When these effects are taken into account, we observe the smear goes to zero as the initial amplitude goes to zero as it should. The simulation code was checked by reverse tracking to reproduce the initial conditions. In $10^{4}$ revolutions, the error in reproducing the initial conditions is $\sim 10^{-13}$, increasing to $\sim 10^{-7}$ in $10^{7}$ revolutions (in double precision on an IBM 3090).

Finally, we also study the effect of tune modulation on the particle motion. The betatron tunes are asumed to be $\nu_{x}=\nu_{x}^{*}+\Delta \nu_{x}$ sin $[n \bar{f}]$ and $\nu_{y}=\nu_{y}^{*}+\Delta \nu_{y}$ sin $[n \bar{f}+\varphi]$ for the n'th revolution and a tune modulation of $\Delta \nu_{s}$ and $\Delta \nu_{y}$. Furthermore, $\bar{f}=2 \pi C f / v$ where $f$ is the frequency of the tune modulation and $\varphi$ sets up a phase difference between the modulations.

\section{THE RESULTS}

The simulation, described above, was used to study the beam-beam interaction. In particular the tunes were varjed from $\nu_{x}^{*}=28.809$ to $\nu_{x}^{*}=28.830$ in steps of 0.0001 and $\nu_{s}^{\circ}=\nu_{x}^{0}-0.004$. Furthermore, $\xi=0.02, \beta=2 \mathrm{~m}$, $\sigma=5.5 \times 10^{-1} \mathrm{~m}, \gamma=100, C=3833.845 \mathrm{~m}, x_{0}=5 \sigma$, $x_{0}^{\prime}=0, y_{0}=5 \sigma$ and $y_{0}^{\prime}=0$. Figure 1 ahow the maximum $E_{x}+E_{y}$ and minimum $E_{x}+E_{j}$ verrus the reaulting $\nu_{x}$, (where $E_{x}$ and $E_{y}$ are the emittances in $\pi$ ram-mrad). In this figure we obeerve 3 major regions: Region (I) show larger emittance to the 16 th order resonances at harmonic 461. Additionally, there are 8 peaks in $\left(E_{x}+E_{y}\right)_{\text {max }}$ and the 8 troughs in $\left(E_{x}+E_{y}\right)_{\min }$ corresponding to the 9 resonances $16 \nu_{x}=461,14 \nu_{x}+2 \nu_{y}=461,12 \nu_{x}+4 \nu_{y}=$ $461, \ldots, 16 \nu_{y}=461$. Also the parametric $16 v_{x}$ and $16 \nu_{y}$ resonances are harder to be recognized. Region II shows no resonances; Region III show some peaks due to the 22nd order resonances with harmonic at 634 .

Although we resonance effects in Fig. 1, long term tracking will not reveal any instabilities or chaotic behavior. When tune modulation effects are included, new effects appear. Consider a tune modulation of $\Delta \nu_{z}=\Delta \nu_{y}=0.001, \varphi=0$ (i.e. tune modulation parallel to the coupling line) and at a frequency of $60 \mathrm{~Hz}$. Note, it take about 1,300 revolutions for the particle to go through one full cycle of the tune modulation, thus, long term tracking is necessary.

Figure 2 plots $\left(E_{z}+E_{y}\right)_{\text {mas }}$ and $\left(E_{z}+E_{y}\right)_{\text {min }}$ versus $\nu_{s}$ with tune modulation on (each point tracbs through $10^{5}$ revolutions). In region $I$, the emittancea have greatly increaned due to the 16th order resonancen. Region III show no significant effect due to the 22nd order rewonance.

The pointe A and B shown on Fig. 2 are the tunes where we performed long term tracking. A particle at point $A$ can crom a 16th order with results shown in Fig.
3. In $10^{6}$ revolutions the resonance can be crossed 1500 times. The motion seems chaotic with large emittance growtti. The total emittance is outside the available aperture. Detailed phase space indicates that the particle is locked on a sum resonance line and then is transported through different resonance to another sum resonance. Through this mechaniom the total emittance is increased irreversibly. For comparison, Fig. 4 shows the tracking in Region II at point B (i.e. point B on Fig. 2). Here, the motion appears to be quite regular and bounded.

\section{CONCLUSION}

In all large colliders such as RHIC, SSC, SPS, Tevatron, etc. it is very likely that these machines will collide beam of unequal sire. In this case, the larger beam can have a significant number of particlea at the position of $5 \sigma$ or larger relative to the amaler beam. Since, these particles may alo have tunes near high order rewonances, they can get lost leading to increased background radiation which the detectors muat be able to handle. However, this will not lead to total beam lom, only losen to particles at the fringes of the beam.

This is expecially important to machines such as RHIC which will collide beame of difierent species. When colliding a proton beam againat a gold beam, the gold beam will be larger. As the tune of the machine croses a high order resonance, there will be an increase in the background of gold ions that the detectore must contend with.

\section{REFERENCES}

[1] L. Evans, "The Beam-Beam Interaction", in Workshop on the RHIC Performance, BNL Report 41604, Pp, 245-251 (1988).

[2] A.W. Chro, "Beam-Beam Instability", AIP Conference Proc. 127, pp. 202- 242 (1983).

[3] A. Geraimov, F. Izrailev, J. Tennywon, A. Tyomnikh, "The Dynamice of the Beam-Beam Interaction", In Lecture Note in Phyaics 247, pp. 154-175 (1985).

[4] S. Myer, "Review of Beam-Beam Simulation", ibid, Pp. 176-237.

[5] A.W. Cheo, P. Bambade, W.T. Weng, "Nonlinear Beam-Beam Resonances", ibid, pp. 77-103.

[6] M. Month, J. Herrere, editora, "Nonlinear Dynamica and the Beam Beam Interaction", AIP Conference Proc. 57, 1979.

[7] S.Y. Lee, S. Tepikian, Tangent Map Analyai of the Beam-Beam Interaction", Third Advanced ICFA Beam Dynamic Workehop, Novosibirck, USSR, pp. 62-65 (1989).

[B] M. Cornacchia, L. Evan, The Effects of Magnetic Nonlinearitien on a Stored Proton Bean and there Implications for Superconducting Storage Ringe". Particle Accelerator 10. pp. 125-144 (1986).

[9] Conceptual Deaign Report for RHIC, BNL Report 52195 (1989). 


\section{DISCLAIMER}

This report was prepared as an account of work sponsored by an agency of the United States Government. Neither the United States Government nor any agency thereof, nor any of their employees, makes any warranty, express or implied, or assumes any legal liability or responsibility for the accuracy, completeness, or usefulness of any information, apparatus, product, or process disclosed, or represents that its use would not infringe privately owned rights. Reference herein to any specific commercial product, process, or service by trade name, trademark, manufacturer, or otherwise does not necessarily constitute or imply its endorsement, recommendation, or favoring by the United States Government or any agency thereof. The views and opinions of authors expressed herein do not necessarily state or reflect those of the United States Government or any agency thereof. 


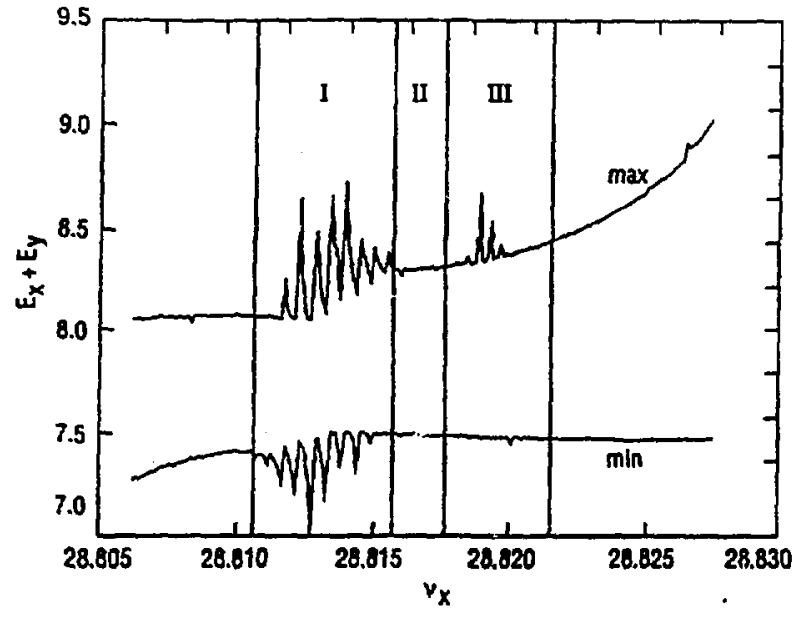

Fig. 1: Plot of $\left(E_{x}+E_{y}\right)_{\min }$ and $\left(E_{x}+E_{y}\right)_{\max }$ versus the hozizontal tune $\nu_{x}$ with no tune modulation. Regions I, II, and III refer to the particle crossing different resonances, see text.

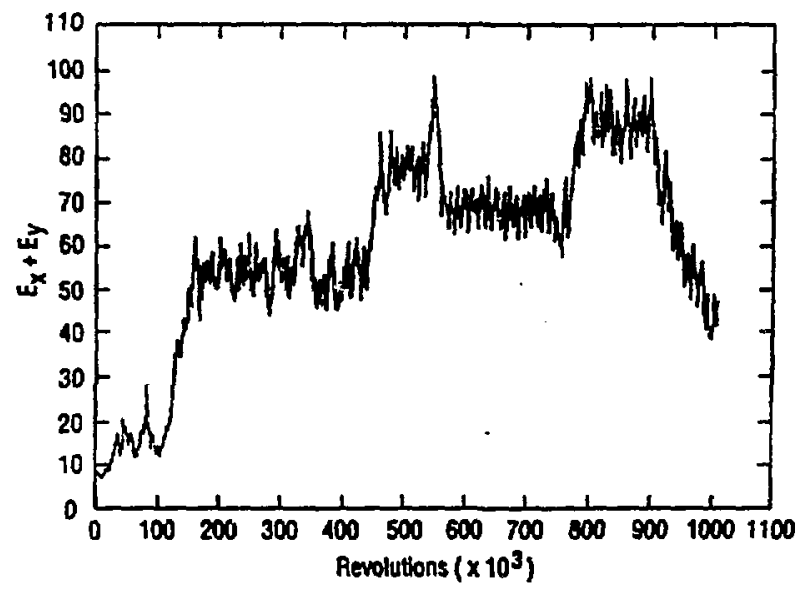

Fig. 3: Long term tracking of particle on a 16th order resonances. The tunes are shown at point $A$ on Fig. 2.

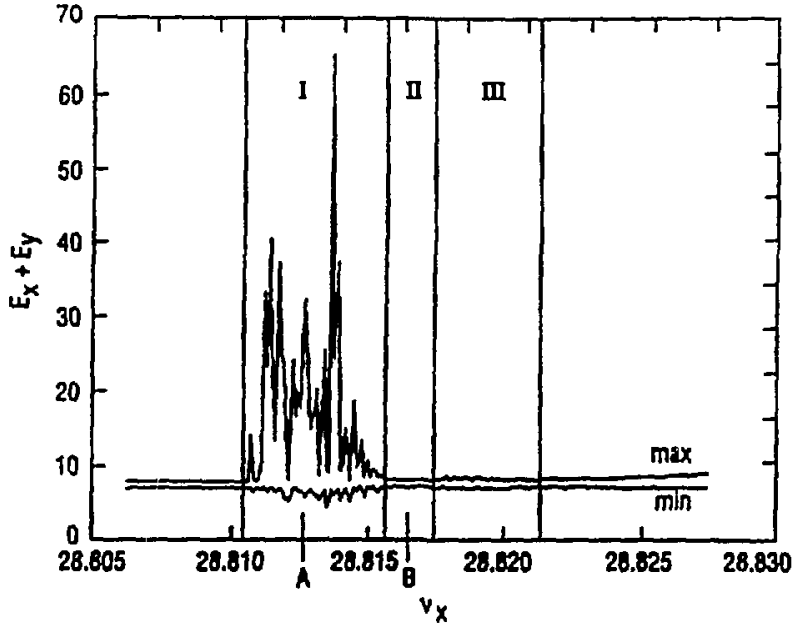

Fig. 2: Plotting $\left(E_{x}+E_{y}\right)_{\min }$ and $\left(E_{x}+E_{y}\right)_{\max }$ versus $\nu_{x}$ with tune modulation. Point $A$ and $B$ refer to Figs. 3 and 4 respectively. Note the expanded scale of the total emittance.

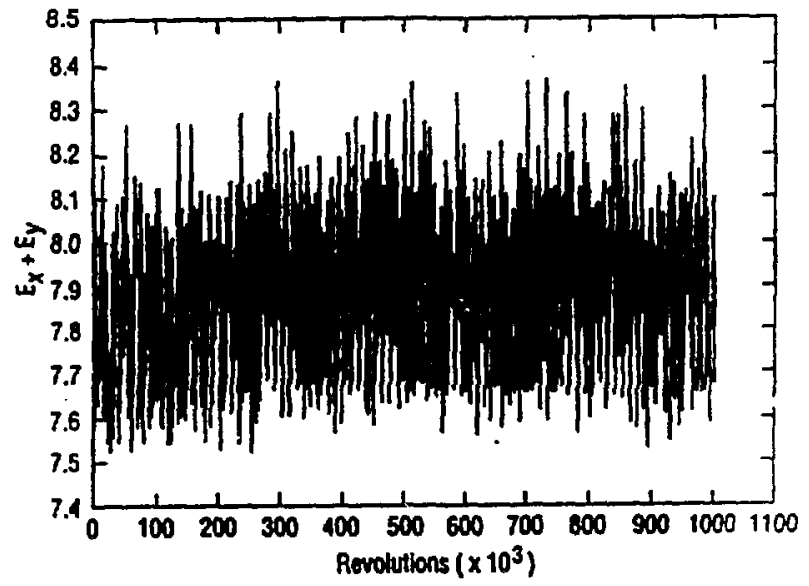

Fig. 4: Long term tracking of a particle away from resonances. The tunes are shown at point B on Fig. 2. 